\title{
Correlation between bone mineral density, isokinetic peak torque and postural balance in postmenopausal women
}

\author{
Laís Campos de Oliveira'; Raphael Gonçalves de Oliveira'; Fabiano Cardoso dos Santos'; \\ Jorge Furtado de Campos Júnior'; Géssika Castilho dos Santos'; Paula Roldão da Silva'; Jadson Marcio da Silva'; \\ Cláudia Roberta Brunnquell Sczepanski'; Berlis Ribeiro dos Santos Menossi'; Fábio Antônio Néia Martini', \\ Luciana da Silva Lirani²; Deise Aparecida de Almeida Pires-Oliveira³.
}

\begin{abstract}
Background: Postmenopausal women typically present loss of bone mineral density (BMD), muscular strength, and postural balance. However, there is no consensus in the literature on the correlation between these variables. Objectives: Our objective was to verify the correlation between BMD, isokinetic peak torque of knee extensors and flexors, and static postural balance in postmenopausal women. Methods: Fifty-one postmenopausal women underwent BMD evaluation through dual-energy X-ray absorptiometry (DXA) for four bone regions (lumbar spine, femoral neck, total hip, and trochanter). Muscular strength of knee extensors and flexors was evaluated in an isokinetic dynamometer at 60 and 180 degrees per second in the dominant lower limb. Static postural balance was evaluated on a force platform during five different tasks (bipodal eyes open, bipodal eyes closed, semi-tandem eyes open, semi-tandem eyes closed, and unipodal). Statistical analysis involved Spearman's correlation coefficient (rho). Results: There was no significant correlation between BMD and isokinetic peak torque of knee extensors and flexors. Approximately half ( 27 of 60 ) of the postural balance variables presented significant inverse correlations $(p<0.05)$ with BMD and of these, the majority (22 of 27) presented moderate correlation (rho between -0.49 and -0.30 ). Most correlations were for bipodal support tasks with eyes open and eyes closed. Conclusion: Greater postural oscillation in different balance tasks was correlated with lower BMD in postmenopausal women. Therefore, it is suggested that intervention protocols for women with impaired BMD, especially osteoporotic, consider balance training as part of rehabilitation.
\end{abstract}

Keywords: Postmenopause, Osteoporosis, Muscle Strength Dynamometer, Postural Balance

\section{BACKGROUND}

Women in the postmenopausal period undergo hormonal changes that contribute to a number of negative health factors, such as reduction in bone mineral density $(\mathrm{BMD})^{(1)}$, loss of muscular strength $^{(2)}$, and lower postural balance ${ }^{(3)}$. These variables, when altered, decrease the functional status and constitute a significant risk factor for an increase in the incidence of falls and a consequent risk of fractures ${ }^{(4)}$, which leads in some cases to morbidity or even mortality ${ }^{(5,6)}$.

However, the literature is inconsistent and inconclusive as to the relation between these variables in postmenopausal women. A few studies have found linear and consistent relations between muscular strength and $\mathrm{BMD}^{(7,8)}$, while the majority have found inconsistent results ${ }^{(9-13)}$. Similarly, when the variable refers to postural balance, a few studies have shown an inverse relationship between greater postural oscillation and $\mathrm{BMD}^{(14,15)}$, while the majority of studies have not identified any relationship ${ }^{(9,11,12,16)}$.
However, to determine correlations between BMD and muscular strength or postural balance in postmenopausal women, some measurement conditions of these variables have not yet been tested, such as the isokinetic peak torque of knee extensors and flexors on an isokinetic dynamometer, or stabilographic analysis of postural oscillation on a force platform under challenging conditions, such as semi-tandem or unipodal support.

Identification of variables correlated with BMD in postmenopausal women becomes important due to the ability to assist professionals in clinical practice regarding treatment priorities for this condition. Thus, the objective of the present study was to identify the correlations between BMD and isokinetic peak torque of knee extensors and flexors and static postural balance in postmenopausal women. We hypothesized that there would be a linear correlation between the variables of isokinetic peak torque and BMD values, while postural balance would be inversely correlated (the greater the postural oscillation, the lower the BMD). 


\section{METHODS}

\section{Sample Selection}

For elaboration of the present cross-sectional study, baseline data from an intervention study were used, approved by the Ethics Committee for Human Beings of the Universidade Norte do Paraná, Brazil (protocol number 1.032.182) and registered in Clinical Trials (NCT02769143). Women who were postmenopausal and without a previous diagnosis of osteopenia or osteoporosis were invited to participate in the sample. For the recruitment, posters were placed in public places, as well as advertisements in printed newspapers, on radios, internet news sites, and newsletters for medical clinics and basic health units, all with local coverage, during the month of May 2016, in the city of Jacarezinho, state of Paraná, Brazil.

The following inclusion criteria were used: clinically confirmed postmenopausal for at least 12 months; not practicing physical exercise for at least six months; the ability to perform activities of daily living without assistance ${ }^{(17,18)}$; presentation of a medical certificate indicating fitness for physical exercise; and cognitive status $\geq 19$ according to the Mini Mental State Exam (MMSE) ${ }^{(19)}$.

Exclusion criteria were: musculoskeletal dysfunctions in the spine or lower limbs in the previous six months; fracture of the spine or lower limbs after 40 years of age; prosthesis in the lower limbs or implants in the spine; secondary causes of bone mass loss; other metabolic bone diseases or diseases that affect bone metabolism; a history of cancer in the previous 5 years; vascular alterations; epilepsy or seizures; cardiac arrhythmia; the use of a pacemaker; cardiorespiratory diseases; diseases in the neuromuscular system; labyrinthitis or vertigo; hospitalization in the previous six months for surgical reasons; thyroid alteration; smoking; use of supplements such as calcium or vitamin D, or containing isoflavones; and the use of medication to increase BMD or to increase muscle mass in the previous 12 months.

During the recruitment period, 51 women between the ages of 40 and 70 met the inclusion/exclusion criteria, who, after being informed about the objectives and methods of the research and prior to any procedure, signed the Free and Informed Consent Term. Next, the volunteers were submitted to standardized evaluation procedures for BMD, isokinetic muscular strength, and static postural balance, with each test performed by the same evaluator, trained and experienced in each procedure.

\section{Evaluation}

Bone mineral density

For bone mass analysis, Dual-energy X-ray Absorptiometry (DXA) equipment Hologic QDR 1000 Plus (Hologic Inc., Waltham, MA, USA) was used. The BMD was expressed in absolute values $\left(\mathrm{g} / \mathrm{cm}^{2}\right.$ - grams of bone mineral content per square centimeter). For classification, the World Health Organization $^{(20)}$ criteria were used, in T-score values: no alteration ( $T>-1)$, osteopenia ( $T$ between -1 and -2.5 ), and osteoporosis $(T<2.5)$. Four different regions were considered: lumbar spine (L1-L4), femoral neck, total hip, and trochanter. In precision studies, the coefficients of variation for this device were $1.3 \%$ for the lumbar spine, $1.4 \%$ for the femoral neck, and $1.2 \%$ for the total hip ${ }^{(21)}$.

\section{Isokinetic peak torque}

The isokinetic peak torque was measured by means of knee extension and flexion movements (concentric/concentric) with Biodex System 4.0 equipment (Biodex Medical Systems, Shirley, NY, USA), using the dominant lower limb, with three attempts of five repetitions each, at angular velocities of 60 and 180 degrees per second $(60 \%$ s and $180 \% \mathrm{~s})$. A 30 second rest interval was respected between attempts ${ }^{(22)}$. For subsequent data analysis, the mean of the three trials was used. Prior to the test, the instrument was calibrated according to the manufacturer's standards. The warm-up was performed for five minutes on a vertical exercise bike, with a light load and at a comfortable speed.

The evaluator then explained the test procedures in detail and positioned the volunteer in the equipment seat with the angle of the hip joint at 120 degrees of flexion. The axis of rotation of the dynamometer arm was aligned with the lateral epicondyle of the femur. The location of force application was positioned approximately two centimeters from the medial malleolus. Belts were attached to the trunk, pelvis, and thigh in order to avoid compensatory movements ${ }^{(23)}$. The volunteers were instructed to perform the maximum voluntary contraction force of the knee extensors, followed immediately by maximal contraction of the knee flexors, in a continuous manner. The words of encouragement "with force" were used in a standardized way throughout the test. For the purposes of analysis, the isokinetic peak torque (PT) was considered, expressed in newtons per meter (N.m).

\section{Postural balance}

To evaluate the static postural balance, a force platform, model BIOMEC400 (EMG System do Brasil Ltda., São Paulo) was used. The evaluator explained the test procedures to the volunteer, who was allowed a short period (approximately 5 minutes) of familiarization with the equipment and the tests to be performed. Subsequently the participants performed the following tasks: bipodal support with eyes open (BP-EO) and bipodal with eyes closed (BP-EC), semi-tandem with eyes open (ST-EO) and semi-tandem with eyes closed (ST-EC), and unipodal (lower dominant limb) with eyes open (UP-EO) (Figure 1). The order of execution of each task (bipodal, semi-tandem, and unipodal) was randomized. 

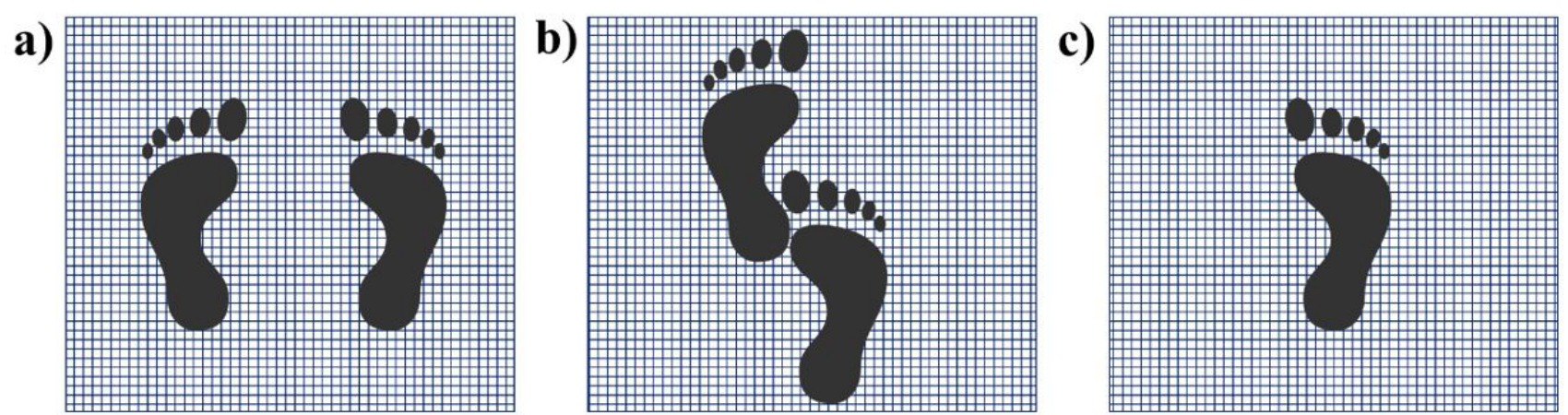

Figure 1. Positioning of the feet on the force platform during postural balance tasks: a) bipodal; b) semi-tandem; c) unipodal.

For each task, three 30-second attempts were performed, with the same rest interval (30 seconds) between them. For data analysis, the mean of the three trials was used. The volunteers were barefoot, with arms loose and relaxed at the side of the body and the cephalic positioning horizontal to the ground plane. All participants stared at a fixed target (the shape of a cross, measuring $15 \mathrm{~cm}$ wide $x 15 \mathrm{~cm}$ high), positioned on the wall, at eye level, at a distance of 2 meters.

Signals of the ground reaction force from the platform measurements were collected at a $100 \mathrm{~Hz}$ sampling. All force signals passed through a Butterworth low pass filter at $35 \mathrm{~Hz}$, after which the signals were converted by means of stabilization analysis, compiled with the MatLab routines from the platform software (The Mathworks, Natick, MA). The calculated balance parameters were: displacement area of the center of pressure (A-COP) expressed in centimeters squared $\left(\mathrm{cm}^{2}\right)$ and mean velocity of oscillation expressed in centimeters per second $(\mathrm{cm} / \mathrm{s})$, in the anteroposterior directions $(A / P)$ and mediolateral directions (M/L). These parameters were chosen as they present good reliability in young and old adults ${ }^{(24)}$.

\section{Statistical Analysis}

Descriptive data are expressed as mean and standard deviation. To verify the normality of the data, the Kolmogorov-Smirnov test was used. Due to the nature of the data, the nonparametric Kruskal-Wallis test was used to compare the variables of peak isokinetic torque and variables of postural balance between the groups formed by BMD classification (no alteration, osteopenia, and osteoporosis) and as post-hoc the Dunn test was used. The absolute measures of each BMD variable (lumbar spine, femoral neck, total hip, and trochanter), expressed in $\mathrm{g} / \mathrm{cm}^{2}$, were correlated with the absolute measures of peak isokinetic torque of knee extensors and flexors and the different variables related to postural balance using the Spearman's correlation coefficient (rho). In relation to the classification of the strength of the correlations, the following values were adopted: $>0.50$ or $<-0.50$ strong correlation; between -0.49 and -0.30 or 0.30 and 0.49 moderate correlation; and values between -0.30 and $<0.30$ weak correlation ${ }^{(25)}$. The confidence interval for all tests was $95 \%(p<0.05)$. Data were processed in the SPSS 20.0 program (SPSS Corp., Chicago, IL, USA).

\section{RESULTS}

\section{Isokinetic peak torque and postural balance between different bone mass classifications}

Table 1 presents the characterization of the sample and the comparisons between the groups formed by the three different BMD classifications (no alteration, osteopenia, and osteoporosis). The Kruskal-Wallis test demonstrated significant differences between the groups for age $(p=0.001)$, with the post-hoc Dunn indicating the groups without alteration ( $p=0.001$ ) and osteopenia $(p=0.016)$, with significantly lower age than the osteoporosis group. Significant differences were found between groups for height $(p=0.010)$, in which the Dunn's post-hoc test indicated that the group with no alteration in BMD presented significantly greater height than the osteoporosis group ( $p=0.007)$. Regarding the measures of postural balance, significant differences were observed for unipodal support with eyes open on the force platform under two conditions: anteroposterior displacement velocity $(p=0.033)$ and mediolateral displacement velocity $(p=0.022)$, with the post-hoc analysis indicating better postural balance ( $p=0.034$ and $p=0.018$, respectively) in the two analyzes for the group without alteration, when compared with the osteoporosis group. Body weight, BMI, isokinetic peak torque of knee extensors and flexors, as well as other variables related to postural balance did not present significant differences between the different BMD classification groups. 
Table 1. Characterization of the sample and comparison of the study variables between the different conditions of bone mineral density in postmenopausal women

\begin{tabular}{|c|c|c|c|c|c|}
\hline Variable & $\begin{array}{c}\text { All } \\
n=51\end{array}$ & $\begin{array}{c}\text { No alteration } \\
n=10\end{array}$ & $\begin{array}{c}\text { Osteopenia } \\
n=31\end{array}$ & $\begin{array}{c}\text { Osteoporosis } \\
n=10\end{array}$ & $\mathbf{p}$ \\
\hline Age (years) & $55.35(6.16)$ & $50.50(5.48)^{\mathrm{a}}$ & $55.13(5.40)^{\mathrm{a}}$ & $60.90(4.84)$ & 0.001 \\
\hline Weight (Kg) & $65.45(7.22)$ & $67.87(5.92)$ & $65.72(7.70)$ & $62.20(6.17)$ & 0.160 \\
\hline Height $(\mathrm{cm})$ & $155.94(5.67)$ & $160.10(6.64)^{\mathrm{a}}$ & $155.94(4.83)$ & $151.80(4.34)$ & 0.010 \\
\hline BMI $\left(\mathrm{Kg} / \mathrm{m}^{2}\right)$ & $26.91(2.57)$ & $26.50(2.03)$ & $27.02(2.88)$ & $26.97(2.16)$ & 0.590 \\
\hline PT extensors (N.m) $60^{\circ} / \mathrm{s}$ & $95.32(21.54)$ & $105.95(25.92)$ & $94.51(21.43)$ & $87.18(13.20)$ & 0.226 \\
\hline PT flexors (N.m) $60^{\circ} / \mathrm{s}$ & 48.78 (14.49) & $54.05(18.64)$ & $47.32(14.47)$ & $48.04(9.06)$ & 0.755 \\
\hline PT extensors (N.m) $180^{\circ} / \mathrm{s}$ & $56.41(11.34)$ & $60.90(13.44)$ & $56.41(11.28)$ & $51.92(8.08)$ & 0.197 \\
\hline PT flexors (N.m) $180^{\circ} / \mathrm{s}$ & $35.59(9.03)$ & $37.80(10.91)$ & $34.73(9.39)$ & $36.06(5.64)$ & 0.774 \\
\hline BP-EO A-COP $\left(\mathrm{cm}^{2}\right)$ & $1.22(0.65)$ & $0.99(0.50)$ & $1.23(0.58)$ & $1.42(0.93)$ & 0.409 \\
\hline BP-EO Velocity A/P (cm/s) & $1.40(0.22)$ & $1.42(0.20)$ & $1.38(0.22)$ & $1.45(0.27)$ & 0.797 \\
\hline BP-EO Velocity M/L $(\mathrm{cm} / \mathrm{s})$ & $1.16(0.17)$ & $1.13(0.21)$ & $1.16(0.17)$ & $1.19(0.16)$ & 0.625 \\
\hline BP-EC A-COP $\left(\mathrm{cm}^{2}\right)$ & $1.36(1.09)$ & $1.17(0.57)$ & $1.27(0.57)$ & $1.81(2.20)$ & 0.850 \\
\hline BP-EC Velocity A/P (cm/s) & $1.52(0.31)$ & $1.54(0.32)$ & $1.49(0.28)$ & $1.59(0.40)$ & 0.816 \\
\hline BP-EC Velocity $\mathrm{M} / \mathrm{L}(\mathrm{cm} / \mathrm{s})$ & $1.19(0.20)$ & $1.15(0.20)$ & $1.19(0.20)$ & $1.24(0.19)$ & 0.600 \\
\hline ST-EO A-COP $\left(\mathrm{cm}^{2}\right)$ & $7.36(4.04)$ & $6.71(3.63)$ & $7.67(4.49)$ & 7.05 (3.09) & 0.888 \\
\hline ST-EO Velocity A/P $(\mathrm{cm} / \mathrm{s})$ & $1.97(0.63)$ & $1.86(0.32)$ & $1.99(0.78)$ & $2.02(0.31)$ & 0.467 \\
\hline ST-EO Velocity M/L (cm/s) & $2.35(0.83)$ & $2.05(0.41)$ & $2.41(0.99)$ & $2.49(0.49)$ & 0.149 \\
\hline ST-EC A-COP $\left(\mathrm{cm}^{2}\right)$ & $9.77(6.27)$ & $8.20(3.21)$ & $9.67(5.12)$ & $11.62(10.67)$ & 0.874 \\
\hline ST-EC Velocity A/P (cm/s) & $2.46(0.71)$ & $2.31(0.51)$ & $2.42(0.76)$ & $2.72(0.73)$ & 0.146 \\
\hline ST-EC Velocity M/L (cm/s) & $3.06(0.92)$ & $2.84(0.73)$ & $3.01(0.91)$ & $3.45(1.07)$ & 0.171 \\
\hline UP-EO A-COP $\left(\mathrm{cm}^{2}\right)$ & $19.93(13.92)$ & $14.42(4.85)$ & $22.96(16.87)$ & $16.05(4.65)$ & 0.094 \\
\hline UP-EO Velocity A/P (cm/s) & $4.29(1.34)$ & $3.52(0.81)^{\mathrm{a}}$ & $4.46(1.54)$ & $4.55(0.74)$ & 0.033 \\
\hline UP-EO Velocity M/L $(\mathrm{cm} / \mathrm{s})$ & $4.94(1.12)$ & $4.25(1.11)^{\mathrm{a}}$ & $4.97(1.07)$ & $5.57(1.01)$ & 0.022 \\
\hline
\end{tabular}

Note: Kruskal-Wallis and Dunn test as post-hoc for comparison between groups (no alteration, osteopenia, and osteoporosis); Data expressed as mean (standard deviation); ${ }^{a}$ different from the osteoporosis group ( $\mathrm{p}<0.05$ ); BMI: body mass index; PT: isokinetic peak torque; N.m: newtons per meter; $60^{\circ} / \mathrm{s}$ : 60 degrees per second; $180^{\circ} / \mathrm{s}: 180$ degrees per second; BP: bipodal support; EO: eyes open; A-COP: displacement area of center of pressure; A/P: anteroposterior displacement; M/L: mediolateral displacement; EC: eyes closed; ST: semi-tandem; UP: unipodal.

\section{Correlations between bone mineral density, isokinetic peak torque, and postural balance}

Table 2 presents the Spearman correlation coefficient (rho) between the absolute values of BMD $\left(\mathrm{g} / \mathrm{cm}^{2}\right)$ and the isokinetic peak torque (N.m) and different postural balance variables measured by the force platform (A-COP, velocity of anteroposterior and mediolateral displacement). Inverse and significant correlations $(p<0.05)$ were found mainly between total hip BMD and different measures of postural balance, with nine of these correlations presenting moderate strength (rho between -0.49 and -0.30 ). Eight postural balance variables also demonstrated inverse and significant correlations $(p<0.05)$ with femoral neck BMD, with four of the correlations showing moderate strength. Despite only six measurements of lumbar spine BMD and three of the trochanter BMD presenting significant and inverse correlations $(p<0.05)$ with postural balance variables, all had moderate strength. Regarding the variables of the isokinetic peak torque of the knee extensors and flexors, no significant correlation $(p>0.05)$ was observed with the BMD of the four bone regions considered. 
Table 2. Correlations between bone mineral density and isokinetic peak torque and postural-static balance in postmenopausal women

\begin{tabular}{|c|c|c|c|c|}
\hline \multirow{2}{*}{ Variable } & \multicolumn{4}{|c|}{ Bone Mineral Density $\left(\mathrm{g} / \mathrm{cm}^{2}\right)$} \\
\hline & Lumbar spine & Femoral neck & Total Hip & Trochanter \\
\hline PT extensors (N.m) $60 \%$ & 0.269 & 0.275 & 0.259 & 0.275 \\
\hline PT flexors (N.m) $60^{\circ} / \mathrm{s}$ & 0.140 & 0.115 & 0.138 & 0.159 \\
\hline PT extensors (N.m) $180^{\circ} / \mathrm{s}$ & 0.221 & 0.259 & 0.246 & 0.209 \\
\hline PT flexors (N.m) $180^{\circ} / \mathrm{s}$ & 0.024 & 0.111 & 0.117 & 0.122 \\
\hline BP-EO A-COP $\left(\mathrm{cm}^{2}\right)$ & -0.184 & $-0.329^{a}$ & $-0.356^{a}$ & -0.256 \\
\hline BP-EO Velocity A/P $(\mathrm{cm} / \mathrm{s})$ & -0.149 & -0.095 & $-0.309^{a}$ & -0.180 \\
\hline BP-EO Velocity M/L $(\mathrm{cm} / \mathrm{s})$ & $-0.311^{a}$ & $-0.291^{a}$ & $-0.411^{b}$ & $-0.342^{\mathrm{a}}$ \\
\hline BP-EC A-COP $\left(\mathrm{cm}^{2}\right)$ & -0.120 & $-0.295^{a}$ & $-0.476^{b}$ & $-0.348^{b}$ \\
\hline BP-EC Velocity A/P $(\mathrm{cm} / \mathrm{s})$ & -0.146 & -0.088 & $-0.330^{a}$ & -0.237 \\
\hline BP-EC Velocity M/L (cm/s) & $-0.317^{a}$ & -0.236 & $-0.449^{b}$ & $-0.391^{b}$ \\
\hline ST-EO A-COP $\left(\mathrm{cm}^{2}\right)$ & -0.006 & $-0.279^{a}$ & -0.244 & -0.169 \\
\hline ST-EO Velocity A/P $(\mathrm{cm} / \mathrm{s})$ & -0.138 & -0.232 & $-0.344^{a}$ & -0.239 \\
\hline ST-EO Velocity M/L $(\mathrm{cm} / \mathrm{s})$ & -0.258 & $-0.366^{b}$ & $-0.312^{a}$ & -0.220 \\
\hline ST-EC A-COP $\left(\mathrm{cm}^{2}\right)$ & -0.101 & -0.090 & -0.098 & -0.025 \\
\hline ST-EC Velocity A/P (cm/s) & $-0.346^{a}$ & -0.233 & $-0.288^{a}$ & -0.147 \\
\hline ST-EC Velocity M/L $(\mathrm{cm} / \mathrm{s})$ & $-0.375^{b}$ & $-0.285^{a}$ & -0.225 & -0.140 \\
\hline UP-EO A-COP $\left(\mathrm{cm}^{2}\right)$ & -0.044 & -0.139 & -0.178 & -0.119 \\
\hline UP-EO Velocity A/P $(\mathrm{cm} / \mathrm{s})$ & $-0.331^{a}$ & $-0.325^{a}$ & $-0.322^{\mathrm{a}}$ & -0.239 \\
\hline UP-EO Velocity M/L $(\mathrm{cm} / \mathrm{s})$ & $-0.364^{b}$ & $-0.325^{a}$ & -0.215 & -0.152 \\
\hline
\end{tabular}

Note: Spearman's correlation test (rho); ${ }^{a} p<0.05 ;{ }^{b} p<0.01$; in bold the correlations with moderate strength (rho between -0.49 and -0.30 ); PT: isokinetic peak torque; N.m: newtons per meter; $60^{\circ} \mathrm{s}$ : 60 degrees per second; $180^{\circ} / \mathrm{s}: 180$ degrees per second; BP: bipodal support; EO: eyes open; A-COP: displacement area of center of pressure; $\mathrm{A} / \mathrm{P}$ : anteroposterior displacement; M/L: mediolateral displacement; EC: eyes closed; ST: semi-tandem; UP: unipodal.

\section{DISCUSSION}

Our results demonstrated that the group with no alterations in BMD presented significantly lower age, greater height, and lower displacement in the unipodal task with eyes open for the anteroposterior and mediolateral velocities when compared to the osteoporosis group. Body weight, BMI, isokinetic peak torque variables, and other variables of postural balance did not demonstrate significant differences between the different BMD classifications. For the correlation tests between the isokinetic peak torque of knee extensors and flexors and BMD, neither of the angular velocities $\left(60 \% \mathrm{~s}\right.$ and $\left.180^{\circ} / \mathrm{s}\right)$ evaluated presented significance. Regarding postural balance and BMD, for almost half the variables ( 27 out of 60 ) there was a significant inverse correlation (the greater the postural oscillation the lower the BMD). Of these, the majority (22 out of 27) presented moderate strength correlations (rho between -0.49 and -0.30 ).
As expected, the group of postmenopausal women diagnosed with osteoporosis had a significantly higher mean age than the osteopenic or unaltered group, corroborating with results presented by other studies, since the advance in age is inversely associated with $\mathrm{BMD}^{(26-28)}$. Regarding the anthropometric variables that characterize the sample, it was observed that women with no alteration in BMD presented significantly greater height than osteoporotic women, however, with no differences for body weight or $\mathrm{BMI}$. A recent systematic review and meta-analysis involving postmenopausal women confirmed that among these three variables, height is the one that is most strongly associated with the diagnosis of osteoporosis, with no evidence for body weight or BMI, which limits interventions as this variable is not modifiable ${ }^{(26)}$.

Contrary to our initial hypothesis, the isokinetic peak torque of knee extensors and flexors at 60\%/s and 180\%/s 
was not significantly different between BMD classifications, nor was there any significant correlation between these variables. In contrast to the present study, Cipriani et al. ${ }^{(7)}$ found significant linear correlations (between 0.307 and 0.354 ) between muscular strength and BMD in postmenopausal women, for all variables analyzed, however, the evaluation involved manual grip strength with a hand dynamometer and BMD was assessed by the proximal radius and phalanges of the last four fingers. In the study by Zhou et al. ${ }^{\left({ }^{8}\right)}$, the isokinetic strength of the flexors and extensors of the right hip and the flexors and extensors of the trunk were evaluated at different angular velocities. The authors identified a significant linear correlation with lumbar spine and femoral neck BMD for the majority of variables analyzed.

Other studies, however, have presented inconsistent or null results. Marin et al. ${ }^{(9)}$ performed different tests of muscular strength (manual grip, elbow extension and flexion with $2 \mathrm{~kg}$ dumbbell, vertical jump, and sit-to-stand test) and significant linear correlations were observed only for the manual grip test in different bone regions in postmenopausal women. Bayramoğlu et al. ${ }^{(13)}$ evaluated the BMD of the lumbar spine, femoral neck, and distal radius. The analyzes of muscular strength involved flexion and extension of the trunk and adduction and abduction of the hip in the isokinetic dynamometer at different angular velocities, in addition to manual handgrip strength. Significant linear correlations were found only for isokinetic strength of the hip abductors and BMD of the femoral neck. In the study by Shin et al. ${ }^{(12)}$ when assessing the strength of knee extensors and flexors with a manual dynamometer, no significant correlation was observed with lumbar spine or femoral neck BMD in postmenopausal women.

In relation to postural balance, the current study found that postmenopausal women with no alterations in BMD demonstrated significantly less body oscillation during the postural balance task in unipodal support (for anteroposterior and mediolateral displacement velocities) when compared with the group that presented osteoporosis. No differences were observed for the other variables. It is noteworthy that among all the tasks of postural equilibrium tested, the unipodal position was the most challenging. This may justify the fact that it was the only one that presented significantly different values between unaltered and osteoporotic women.

Until now, other studies that have attempted to identify differences in the postural balance of postmenopausal women between BMD classifications by means of stability platform analysis did not test the unipodal support condition. Cangrussu et al. ${ }^{(16)}$ did not find significant differences between women with and without osteoporosis, for any variable of the force platform in bipodal support with eyes open. However, Burke et al. ${ }^{(15)}$ identified significantly higher postural oscillation in the eyes open task on the force platform on a stable surface in the group of women with osteoporosis. It is notable that they did not observe significant differences in the more challenging task (unstable surface). Likewise, Hita-Contreras et al. ${ }^{(14)}$ when testing different conditions on the force platform, observed a significant difference between the groups with and without osteoporosis, only for anteroposterior displacement with eyes open, in which the group with osteoporosis presented greater postural oscillation.

In the current study, correlations between postural oscillation in the different balance tasks and BMD values of four bone regions were also identified; in this case; in approximately half the variables, significant and inverse correlations were observed. The bone region that presented the highest number of correlations for the different body positions during the postural balance tests was the total hip. However, for all five tasks tested (bipodal with eyes open, bipodal with eyes closed, semi-tandem with eyes open, semi-tandem with eyes closed, and unipodal with eyes open) significant correlations were observed with different bone regions. The tasks of postural balance that involved a greater number of significant correlations refer to bipodal support with eyes open and eyes closed, each on seven occasions.

Impairment in postural balance in people with lower BMD may be associated with the fact that bone demineralization affects the skeletal system, including the temporal bone, which contains the cochlea and the vestibular labyrinth. It has been demonstrated in a representative sample of adults that individuals of 40 years of age or older who present a decrease in BMD are twice as likely to present postural balance impairment, increasing to approximately fourfold among those aged $\geq 65$ years $^{(29)}$.

However, among studies that have so far sought to correlate postural balance and BMD variables in postmenopausal women, only Marin et al. ${ }^{(9)}$ tested unipodal support for 30 seconds with eyes open, without the use of equipment, and found a weak significant linear correlation $(r=0.24)$ between less time in the test and lumbar spine BMD, with no differences for other bone regions. While the study by Shin et al. ${ }^{(12)}$, when testing two tasks on the force platform (bipodal support with eyes open and bipodal support with eyes closed) did not find significant correlations with the lumbar spine and femoral neck BMD. Likewise, Edwards et al. ${ }^{(11)}$ testing dynamic balance in a three-meter gait test, found no association with BMD of the tibia and radium in postmenopausal women.

Considering the contradictory results found by the different studies, both for variables involving muscular strength and postural balance in relation to BMD in postmenopausal women, further studies should be performed. However, considering that greater postural oscillation significantly increases the incidence of falls and consequent risk of fracture in postmenopausal women ${ }^{(4)}$; and due to the results of the present study, it is suggested that interventions involving the rehabilitation of osteoporotic women consider postural balance training, in order to minimize the occurrence of falls in a population exposed to a higher risk of fracture due to lower BMD. 


\section{Strengths and Limitations}

This was the first study to verify the correlations between isokinetic peak torque of knee extensors and flexors and BMD in postmenopausal women. Furthermore, it was the first to test two different challenging conditions (semi-tandem and unipodal support) for static postural balance, seeking correlations with BMD. Thus, the present study contributes to discussions on the subject, in order to assist professional interventions with women with BMD impairment, such as osteopenic and, mainly, osteoporotic, and to advance discussions capable of contributing to future studies.

As limitations, the transversal nature of the study stands out, preventing any causal inference. The small sample number is also a limitation, considering that the sample was derived from the baseline of a clinical trial, it was not possible to perform a sample calculation specifically involving the variables analyzed herein. However, as the inclusion/exclusion criteria were rigorous, the sample of the current study can be considered homogeneous, which increases the internal validity of the presented data, although, to some extent, limits the external validity.

\section{CONCLUSION}

Postmenopausal women with different BMD classifications (unaltered, osteopenic, and osteoporotic) did not present significant differences in isokinetic peak torque values of knee extensors and flexors, in addition, correlations were not observed between isokinetic peak torque and BMD of different regions. On the other hand, women with no alterations in BMD presented lower oscillation of postural balance for unipodal support tasks when compared with osteoporotic women. In addition, different static postural balance tasks demonstrated a significant inverse correlation with BMD (higher postural oscillation correlated with lower BMD) in postmenopausal women. Thus, interventions involving the rehabilitation of osteoporotic women should consider postural balance exercises, since this variable may be significantly compromised and correlated with BMD.

\section{AUTHORS' CONTRIBUTIONS}

LCO, RGO and DAAPO elaborated the study design. FCS, JFCJ, GCS, PRS and JMS performed the data collection. RGO and LCO performed the statistical analysis. CRBS, BRSM, FANM and LSL were involved in the preparation of the manuscript. LCO, RGO and DAAPO performed the critical intellectual revision of the manuscript. All authors read and approved the final manuscript.

\section{CONFLICTS OF INTEREST}

The authors declare that they have no conflicts of interest in the research.

\footnotetext{
AUTHOR DETAILS

2 Universidade Federal do Vale do São Francisco - UNIVASF, Petrolina - PE, Brasil

${ }^{3}$ Centro Universitário de Anápolis - UniEVANGÉLICA, Anápolis - GO, Brasil
}

\section{REFERENCES}

1. Eastell R, O'Neill TW, Hofbauer LC, Langdahl B, Reid IR, Gold DT, et al. Postmenopausal osteoporosis. Nat Rev Dis Primers 2016; 2: 16069.

2. Maltais ML, Desroches J, Dionne IJ. Changes in muscle mass and strength after menopause. J Musculoskelet Neuronal Interact 2009; 9: 186-197.

3. Mikó I, Szerb I, Szerb A, Poor G. Effectiveness of balance training programme in reducing the frequency of falling in established osteoporotic women: A randomized controlled trial. Clin Rehabil 2017; 31: 217-224.

4. Drozdzowska B, Wiktor K, Pluskiewicz W. Functional status and prevalence of falls and fractures in population-based sample of postmenopausal women from the RAC-OST-POL Study. Int J Clin Pract 2013; 67: 673-681.

5. Afrin N, Honkanen R, Koivumaa-Honkanen H, Lukkala P, Rikkonen T, Sirola J, et al. Multimorbidity predicts falls differentially according to the type of fall in postmenopausal women. Maturitas 2016; 91: 19-24.

6. Sattui SE, Saag KG. Fracture mortality: associations with epidemiology and osteoporosis treatment. Nat Rev Endocrinol 2014; 10: 592-602.

7. Cipriani C, Romagnoli E, Carnevale V, Raso I, Scarpiello A, Angelozzi $M$, et al. Muscle strength and bone in healthy women: effect of age and gonadal status. Hormones (Athens) 2012; 11: 325-332.

8. Zhou Z, Zheng L, Wei D, Ye M, Li X. Muscular strength measurements indicate bone mineral density loss in postmenopausal women. Clin Interv Aging 2013; 8: 1451-1459.

9. Marin RV, Pedrosa MA, Moreira-Pfrimer LD, Matsudo SM, LazarettiCastro M. Association between lean mass and handgrip strength with bone mineral density in physically active postmenopausal women. J Clin Densitom 2010; 13: 96-101.

10. Ahedi H, Aitken D, Scott D, Blizzard L, Cicuttini F, Jones G. The association between hip muscle cross-sectional area, muscle strength, and bone mineral density. Calcif Tissue Int 2014; 95: 64-72.

11. Edwards MH, Gregson CL, Patel HP, Jameson KA, Harvey NC, Sayer AA. Muscle size, strength, and physical performance and their associations with bone structure in the Hertfordshire Cohort Study. J Bone Miner Res 2013; 28: 2295-2304.

12. Shin S, Lee K, Song C. Relationship of body composition, knee extensor strength, and standing balance to lumbar bone mineral density in postmenopausal females. J Phys Ther Sci 2016; 28: 2105-2109.

13. Bayramoğlu M, Sözay S, Karataş M, Kilinç $S$. Relationships between muscle strength and bone mineral density of three body regions in sedentary postmenopausal women. Rheumatol Int 2005; 25: 513-517.

14. Hita-Contreras F, Martínez-López E, González-Matarín P, Mendoza N, Cruz-Díaz D, Ruiz-Ariza A, et al. Association of bone mineral density with postural stability and the fear of falling in Spanish postmenopausal women. Maturitas 2014; 79: 322-328.

15. Burke TN, França FJ, Meneses SR, Cardoso VI, Pereira RM, Danilevicius CF. Postural control among elderly women with and without osteoporosis: is there a difference? Sao Paulo Med J 2010; 128: 219-224.

16. Cangussu LM, Nahas-Neto J, Petri Nahas EA, Rodrigues Barral AB, Buttros Dde A, Uemura G. Evaluation of postural balance in postmenopausal women and its relationship with bone mineral density--a cross sectional study. BMC Musculoskelet Disord 2012; 13:2.

17. Katz S, Ford A, Moskowitz RW, Jackson BA, Jaffe MW. Studies of illness in the aged. The index of ADL: a standardized measure of biological and psychosocial function. JAMA 1963; 21: 914-919.

18. Lawton MP, Brody EM. Assessment of older people: self-maintaining and instrumental activities of daily living. Gerontologist 1969; 9: 179-186

19. Folstein MF, Folstein SE, McHugh PR. "Mini-mental state". A practical method for grading the cognitive state of patients for the clinician. J Psychiatr Res 1975; 12: 189-198.

20. World Health Organization. Prevention and management of osteoporosis. Genebra: WHO; 2003. 
21. Tothill $P$, Hannan WJ. Precision and accuracy of measuring changes in bone mineral density by dual-energy X-ray absorptiometry. Osteoporos Int 2007; 18: 1515-1523.

22. Bottaro M, Russo AF, de Oliveira RJ. The effects of rest interval on quadriceps torque during an isokinetic testing protocol in elderly. J Sports Sci Med 2005; 4: 285-290.

23. Stumbo TA, Merriam S, Nies K, Smith A, Spurgeon D, Weir JP. The effect of hand-grip stabilization on isokinetic torque at the knee. J Strength Cond Res 2001; 15: 372-377.

24. da Silva RA, Bilodeau M, Parreira RB, Teixeira DC, Amorim CF. Age-related differences in time-limit performance and force platform-based balance measures during one-leg stance. J Electromyogr Kinesiol 2013; 23: 634-639.

25. Cohen J. A power primer. Psychol Bull 1992; 112: 155-159.
26. Thulkar J, Singh S, Sharma S, Thulkar T. Preventable risk factors for osteoporosis in postmenopausal women: Systematic review and metaanalysis. J Midlife Health 2016; 7: 108-113.

27. Runolfsdottir HL, Sigurdsson G, Franzson L, Indridason OS. Gender comparison of factors associated with age-related differences in bone mineral density. Arch Osteoporos 2015; 10: 214.

28. Hung VW, Zhu TY, Cheung WH, Fong TN, Yu FW, Hung LK, et al. Age-related differences in volumetric bone mineral density, microarchitecture, and bone strength of distal radius and tibia in Chinese women: a highresolution pQCT reference database study. Osteoporos Int 2015; 26 : 1691-1703.

29. Mendy A, Vieira ER, Albatineh AN, Nnadi AK, Lowry D, Gasana J. Low bone mineral density is associated with balance and hearing impairments. Ann Epidemiol 2014; 24: 58-62. 